\title{
BÁO CÁO KỸ THUẬT: MỤC TIÊU PHÁT TRIỂN BỀN VŨ̉NG \\ NĂNG LỰC SỐ CHO GIÁO DỤC MẦM NON (0 5 tuổi)
}

\author{
Ngô Huy Tâm ${ }^{1}$, Hoàng Anh Đức ${ }^{2}$ \\ ${ }^{1}$ Trường PTLC Phenikaa
}

${ }^{2}$ Trung tâm Nghiên cứu và Phát triển Giáo dục EdLab Asia

\begin{tabular}{|c|c|}
\hline \multicolumn{2}{|r|}{ NộI DUNG CHÍNH } \\
\hline Đề mục & Nội dung chi tiết \\
\hline \multirow{2}{*}{ I. Khái quát chung } & $\begin{array}{l}\text { Xu hướng tất yếu của thời đại: Sự phổ biến của các thiết bị thông minh và } \\
\text { việc sử dụng các thiết bị thông minh ở trẻ nhỏ }\end{array}$ \\
\hline & $\begin{array}{l}\text { Mối lo ngại về ảnh hưởng tiêu cực của các thiết bị thông minh đến sự phát } \\
\text { triển thể chất và tinh thần của trẻ nhỏ }\end{array}$ \\
\hline \multirow{2}{*}{$\begin{array}{l}\text { II. Các nghiên cứu } \\
\text { về "Năng lực kỹ } \\
\text { thuật số" (digital } \\
\text { literacy) }\end{array}$} & $\begin{array}{l}\text { 1. Tổng hợp các khái niệm/định nghĩa về "Năng lực kỹ thuật số” được đưa } \\
\text { ra bởi các nghiên cứu lớn trên thế giới }\end{array}$ \\
\hline & 2. Tổng quan một số khung năng lực "Năng lực kỹ thuật số ở trẻ" hiện nay \\
\hline \multirow{4}{*}{$\begin{array}{l}\text { III. Các nội dung } \\
\text { quan trọng trong } \\
\text { tiếp cận kỹ thuật } \\
\text { số ở trẻ }\end{array}$} & $\begin{array}{l}\text { 1. Các khuyến cáo và hướng dẫn y khoa về sức khỏe của trẻ khi tiếp xúc } \\
\text { với các thiết bị điện tử }\end{array}$ \\
\hline & 2. Mối liên hệ giữa kỹ thuật số và sự phát triển nhận thức, ngôn ngữ của trẻ \\
\hline & 3. Mối liên hệ giữa kỹ thuật số và sự phát triển cảm xúc - xã hội của trẻ \\
\hline & 4. An toàn trên không gian số ở trẻ nhỏ \\
\hline $\begin{array}{l}\text { IV. Một số gợi ý } \\
\text { định hướng }\end{array}$ & Mục tiêu phát triển bền vững Năng lực số cho giáo dục mầm non \\
\hline
\end{tabular}




\section{Khái quát chung}

Cùng với sự phát triển của khoa học công nghệ hiện đại, trẻ nhỏ (từ 0-5 tuổi) ngày nay đang sống trong một môi trường bao quanh bởi các thiết bị điện tử thông minh như điện thoại, máy tính bảng, laptop... Ngày càng có nhiều trẻ, ngay trong các gia đình không quá khá giả, cũng được tiếp cận với điện thoại thông minh ${ }^{1}$. Mặc dù công nghệ đem đến nhiều lợi ích to lớn, việc trẻ nhỏ sớm được tiếp xúc với các thiết bị này cũng đặt ra nhiều băn khoăn liên quan đến sức khỏe thể chất và tinh thần của trẻ, bởi đây là giai đoạn tối quan trọng khi bộ não phát triển với tốc độ rất nhanh và mạnh mẽ².

\section{Các nghiên cứu về "Năng lực kỹ thuật số" (digital literacy)}

1. Tổng hợp các khái niệm/dịnh nghĩa về "Năng lục kỹ thuật số" đuợc đưa ra bởi các nghiên cứu lớn trên thế giới

"Năng lực kỹ thuật số” được nhắc đến trong những nghiên cứu khác nhau với nhiều tên gọi như: “digital literacy” (trình độ kỹ thuật số), "digital skills" (kỹ năng kỹ thuật số), hay "digital competences” (năng lực kỹ thuật số). Ngoài ra, việc định nghĩa cụm từ này cũng rất đa dạng, và được đưa ra bởi nhiều tổ chức lớn và uy tín trên thế giới như UNESCO, Trường Kinh tế London, Ủy ban Châu Âu, Hội đồng Châu Âu, Văn phòng UNESCO khu vực Châu Á Thái Bình Dương, Viện DQ...

Dù có những sự khác biệt nhất định trong cách định nghĩa, "năng lực kỹ thuật số" có thể được hiểu là: "Khả năng truy cập, quản lý, hiểu, tích hợp, giao tiếp, đánh giá và tạo ra thông tin một cách an toàn và hợp lý thông qua các công nghệ số nhằm phục vụ các mục đích học tập, nghiên cứu, giải trí, và các mục đích làm việc chuyên nghiệp khác. Trình độ kỹ thuật số cần thiết gắn liền với trình độ về máy tính, trình độ công nghệ thông tin, trình độ thông tin số và các phương tiện truyền thông"3.

\section{Tổng quan một số khung năng lục "Năng lục kỹ thuật số ở trẻ” hiện nay}

Nhiều khung "Năng lực kỹ thuật số" đã được xây dựng bởi các tổ chức lớn và uy tín chẳng hạn:

- Chương trình Úc v8.3 của Cơ quan báo cáo và đánh giá chương trình giảng dạy của Úc

- Chương trình Công dân Số của Common Sense Education

- Dự án DQ bởi Viện DQ 
- Các nghiên cứu về Khung Tham chiếu Toàn cầu về Các kỹ năng trình độ Kỹ thuật số của UNESCO

- Khung DigComp của Ủy ban Châu Âu

- Khung Trình độ Thông tin và Đa phương tiện của UNESCO

- Khung Giáo dục Công dân số của Hội đồng Châu Âu

- Khung Nghiên cứu kỹ năng

- Khung Trí tuệ số của Tổ chức Hợp tác và Phát triển Kinh tế

- Mô hình năng lực số của Công ty Jisc (Anh)

- Chương trình Learning Wales hoặc Chương trình năng lực số British Columbia

UNESCO đã tổng hợp và phân tích 13 khung "năng lực kỹ thuật số" khác nhau4. Mặc dù có sự khác biệt về cách tiếp cận, thể hiện và độ tuổi nhắm đến, các khung này có một số điểm tương đồng, chẳng hạn:

- Quản lý thời gian tiếp xúc màn hình (screen time management)

- An toàn và bảo mật số (safety and security)

- Năng lực kỹ thuật số (digital literacy)

- Cộng tác và giao tiếp, truyền thông (collaboration and communications)

- Nội dung số và sáng tạo (digital content and creativity)

\section{CÁC NỘI DUNG QUAN TRỌNG TRONG TIẾP CẬN KỸ THUẬT SỐ Ở TRẺ EM}

Khi xét đến việc tiếp cận kỹ thuật số trong trẻ em từ 0-5 tuổi, nhất thiết phải xem xét đến các khuyến cáo và hướng dẫn y khoa, bởi đây là giai đoạn hết sức nhạy cảm và trẻ rất dễ bị tổn thương cả về sức khỏe thể chất và tinh thần. Do đó, tiếp cận kỹ thuật số ở trẻ em có được thể tổng hợp lại thành một số thông tin khái quát như sau:

- Các khuyến cáo và hướng dẫn y khoa về sức khỏe của trẻ khi tiếp xúc với các thiết bị điện tử

- Năng lực số trong sự phát triển nhận thức, ngôn ngữ của trẻ

- Năng lực số trong sự phát triển cảm xúc - xã hội của trẻ

- Một số khuyến nghị khác cho trẻ trong thời đại số (an toàn, bảo mật...) 


\section{Nội dung chi tiết}

1. Các khuyến cáo và huoóng dẫn y khoa về sức khỏe của trẻ khi tiếp xúc với các thiết bị điện tư

Trẻ dưới 2 tuổi cần những trải nghiệm thực tế và tương tác xã hội với người chăm sóc tin cậy để phát triển nhận thức, ngôn ngữ, vận động, và các kỹ năng xã hội, điều mà các nội dung số không thể đem lại ${ }^{5}$.

Bên cạnh đó, cho trẻ thường xuyên sử dụng các thiết bị thông minh đồng nghĩa với việc tạo nên thói quen ít vận động (sedentary), điều này có nguy cơ dẫn đến một số vấn đề sức khỏe và phát triển như:

- Thừa cân béo phì. Việc sử dụng các thiết bị thông minh với cường độ cao ở trẻ nhỏ có liên quan đến việc tăng chỉ số $\mathrm{BMI}^{6}$, và có thể giải thích cho việc chênh lệch nguy cơ béo phì ở một số trẻ7; đồng thời tạo tiền đề cho việc tăng cân sau này ${ }^{8}$.

- Giấc ngủ. Gia tăng tiếp xúc với màn hình tivi, máy tính hay điện thoại trong phòng ngủ từ nhỏ có mối liên quan đến thời lượng ngủ ít hơn vào ban đêm của trè9. Nguyên lý của mối liên quan này là bởi những nội dung kích thích trẻ ${ }^{10}$ và việc ức chế melatonin nội sinh do ánh sáng xanh phát ra từ màn hình ${ }^{11}$.

Do vậy, để đảm bảo sức khỏe thể chất của trẻ không bị ảnh hưởng tiêu cực, cha mẹ và người chăm sóc cần tuân thủ các hướng dẫn y khoa khi cho trẻ tiếp cận với kỹ năng số, chẳng hạn ${ }^{12}$ :

- Trẻ sơ sinh dưới 1 tuổi: không nên tiếp xúc với màn hình điện tử.

- Trẻ 1 tuổi: không hoặc tiếp xúc rất ít. Đây là giai đoạn phát triển quan trọng của trẻ, trẻ cần được khuyến khích hoạt động thể chất và tương tác sáng tạo với người xung quanh nhiều nhất có thể.

- Trẻ 2 tuổi: Tổng thời lượng tiếp xúc không quá 1 giờ. Nếu trẻ tiếp xúc với các nội dung số, cha mẹ hoặc người chăm sóc cần xem và tương tác cùng trẻ.

- Trẻ 3-4 tuổi: tối đa không quá 1 giờ/ngày. Khi cho trẻ xem, cha mẹ cần kết hợp đọc và kể chuyện cho trẻ.

2. Năng lực số trong sự phát triển nhận thức của trẻ 
Nhắc đến nhận thức là nhắc đến sự phát triển não bộ. Nhận thức là một thuật ngữ chung bao gồm các quá trình tư duy như tập trung, hiểu, tiếp nhận, ghi nhớ, giải quyết vấn đề. Cần lưu ý rằng việc phát triển các chức năng nhận thức của não bộ gắn liền với các chuyển động của cơ thể và các hoạt động vật lý.

Trong khi đó, việc trẻ tiếp xúc với các thiết bị số đồng nghĩa với việc thời gian hoạt động của trẻ bị giảm đi. Nghiên cứu chỉ ra tivi, video games... đã làm giảm khả năng tập trung của trẻ, tiếp nhận và phân tích thông tin. Điều này ảnh hưởng đến sự phát triển của bộ não trẻ trong việc tiếp nhận thông tin ${ }^{13}$.

Do đó, vấn đề cấp thiết đặt ra là tạo môi trường vận động cho trẻ dưới các hình thức phong phú đa dạng. Trẻ được vận động sẽ có cơ hội phát triển não bộ tốt hơn, toàn diện hơn.

\section{Năng lực số trong sự phát triển ngôn ngũ của trẻ}

Nhiều nghiên cứu về mối liên quan giữa việc tiếp xúc với các thiết bị điện tử (tivi, điện thoại, máy tính) và sự phát triển ngôn ngữ của trẻ nhỏ được thực hiện, có thể tổng hợp lại một số phát hiện đáng chú ý như sau:

- Việc phát triển ngôn ngữ ở trẻ nhỏ có thể bị ảnh hưởng bởi các đồ chơi số bằng việc giảm giao tiếp với bố mẹ ${ }^{14}$. Kết luận này được đưa ra sau khi quan sát người mẹ và con trong 2 lần: 1 lần với đồ chơi số và 1 lần với đồ chơi truyền thống. Khi chơi với sách điện tử, giao tiếp giữa mẹ và con ít hơn. Những đặc điểm nhất định của đồ chơi số có thể cản trở trẻ và mẹ chơi và giao tiếp, điều này có hại đối với sự phát triển của trẻ vì trẻ không giao tiếp bằng lời nhiều nữa.

- Tuy nhiên, cũng có nghiên cứu kết luận điều ngược lại ${ }^{15,16}$. Đó là khi ngôn ngữ tương tác của trẻ được bởi kích thích và mở rộng bởi các đồ chơi điện tử, các thiết bị công nghệ như điện thoại, máy tính có các nội dung tính năng nhất định phù hợp với lứa tuổi ${ }^{14}$. Những trẻ em trong nghiên cứu này được tiếp xúc nhiều hơn và đã phát triển các kiến thức công nghệ khi bắt đầu đi học, đặc biệt là các trải nghiệm giao tiếp ${ }^{15}$. Như vậy, nếu công nghệ cho trẻ em được phát triển phù hợp với độ tuổi, thì nó có thể hỗ trợ phát triển ngôn ngữ cho trẻ nhỏ ${ }^{14}$.

4. Năng lực số trong sụ̣ phát triển cảm xúc-xã hội của trẻ 
Phát triển cảm xúc-xã hội cũng là một phần quan trọng trong sự phát triển của trẻ. Chúng ta đều biết rằng sự phát triển cảm xúc của trẻ có liên quan mật thiết đến sự phát triển xã hội. Cha mẹ có thể cho trẻ tiếp xúc với các thiết bị và nội dung số trong một khoảng thời gian ngắn, tuy nhiên, khi các thiết bị và nội dung số bắt đầu thay thế tương tác người với người, là lúc phát sinh các vấn đề. Một số nghiên cứu đã ghi lại những tác động xã hội tiêu cực từ thời gian sử dụng máy tính. Trẻ đang phát triển cần thời gian tương tác trực tiếp với mọi người thay vì tương tác công nghệ để phát triển và đạt được các mốc về vận động ${ }^{13}$. Jenny Radesky, giảng viên lâm sàng về phát triển-hành vi nhi khoa tại Trường Đại học Y Boston, kêu gọi các bậc cha mẹ tăng cường "tương tác trực tiếp" với con cái. Cô khuyến khích sự tương tác gia đình "không kết nối mạng" nhiều hơn nói chung và gợi ý rằng trẻ nhỏ có thể được hưởng lợi từ "giờ sinh hoạt gia đình" có chất lượng mà không cần bất kỳ thiết bị di động và truyền hình nào ${ }^{18}$.

\section{An toàn trên không gian số ở trẻ nhỏ}

Trẻ em là nhóm đối tượng dễ bị tổn thương nhất trên không gian mạng. Trong bối cảnh mạng xã hội và các thiết bị di động phát triển mạnh mẽ, việc trẻ bị lợi dụng trở thành nạn nhân, mục tiêu của các đối tượng bắt nạt, xâm hại tình dục, buôn người... càng trở nên phổ biến. Trẻ có thể truy cập dễ dàng các trang web không an toàn, cùng sự hiểu biết hạn chế của trẻ và chính cha mẹ, khiến vấn đề về an toàn trên không gian số càng trở nên cấp thiết.

Các nhà nghiên cứu phân loại các nguy cơ trên không gian số thành 3 nhóm ${ }^{19}$ :

- Nội dung (content): Trẻ tiếp xúc với nội dung không phù hợp như khiêu dâm, tình dục, bạo lực, quảng cáo, phân biệt chủng tộc, nội dung thù ghét...

- Liên lạc (contact): Trẻ tham gia vào một môi trường không an toàn, chẳng hạn bị người lớn liên lạc và dụ dỗ cho mục đích tình dục hoặc các hành vi có nguy cơ

- Thực hiện (conduct): Trẻ cư xử và góp phần vào một nội dung hoặc liên lạc có nguy cơ, chẳng hạn trẻ viết hoặc tạo các nội dung thù ghét với các trẻ khác, đăng ảnh khiêu dâm...

\section{MộT SỐ GỢI Ý ĐỊNH HƯớNG}

Sau khi review các nghiên cứu liên quan đến năng lực số, an toàn số cho trẻ em, có thể rút ra một số điều tổng kết như sau:

- Các nghiên cứu có sự đa dạng về nhóm tuổi trẻ em, tuy nhiên, đối với nhóm trẻ 0-5 tuổi, nội dung chính hướng đến là về sức khỏe thể chất và tinh thần, an toàn trên không gian số 
- Bên cạnh đó, hầu như không có một khung năng lực số nào cho trẻ em ở độ tuổi này, bởi một số nguyên nhân quan trọng như:

+ Trẻ ở độ tuổi 0-5 có đặc điểm là phát triển không đồng đều. Do đó, các nhà khoa học trẻ nhỏ và bác sĩ nhi khoa thường chỉ đưa ra các Mốc phát triển về thể chất, tinh thần và cảm xúc ở từng giai đoạn, để phụ huynh có cơ sở tham khảo.

+ Độ tuổi này ưu tiên về phát triển thể chất, trong khi đó các hoạt động liên quan đến thiết bị điện tử thuộc nhóm hoạt động ít vận động, điều này không được khuyến khích trong khoa học phát triển trẻ nhỏ.

Với những tổng kết trên, nhóm nghiên cứu có một số đề xuất như dưới đây:

- Không xây dựng “Khung năng lực số” cho trẻ ở độ tuổi 0-5 với mục đích đánh giá, kiểm tra.

- Thay vào đó, xây dựng "Khung tiếp cận năng lực số", với trọng tâm là các "Nhóm mục tiêu phát triển bền vững năng lực số cho giáo dục mầm non” để cha mẹ, thầy cô, các trường học, các tổ chức giáo dục có thể tham khảo, định hướng và phối hợp định hướng cho trẻ.

- Đề xuất các nội dung chính trong "Nhóm mục tiêu phát triển bền vững năng lực số cho giáo dục mầm non" gồm các nhóm mục tiêu sau, với chủ thể thụ hưởng là học sinh mầm non:

+ Cuộc sống số khỏe mạnh

+ Năng lực số và phát triển nhận thức

+ Năng lực số và phát triển ngôn ngữ

+ Năng lực số và phát triển cảm xúc-xã hội

+ Cuộc sống số an toàn

+ Công bằng và bình đẳng trong phát triển năng lực số 


\section{MỤC TIÊU PHÁT TRIỄN BỄN VŨ̉NG \\ NĂNG LỰC SỐ CHO GIÁO DỤC MẦM NON}

\begin{tabular}{|c|c|c|}
\hline Nhóm mục tiêu & Mục tiêu & TLTK \\
\hline \multirow[t]{2}{*}{ 1. Cuộc sống số khoẻ mạnh } & $\begin{array}{l}\text { 1.1. Đảm bảo sức khỏe thể chất trong môi } \\
\text { trường số }\end{array}$ & \multirow[t]{2}{*}{$\begin{array}{l}{[20],[21],} \\
{[25]}\end{array}$} \\
\hline & $\begin{array}{l}\text { 1.2. Đảm bảo sức khỏe tinh thần trong môi } \\
\text { trường số }\end{array}$ & \\
\hline \multirow[t]{2}{*}{$\begin{array}{l}\text { 2. Năng lực số và phát triển } \\
\text { nhận thức }\end{array}$} & $\begin{array}{l}\text { 2.1. Đảm bảo sự phát triển nhận thức của trẻ } \\
\text { trong môi trường số }\end{array}$ & \multirow[t]{2}{*}{ [20], [24] } \\
\hline & $\begin{array}{l}\text { 2.2. Xây dựng năng lực tư duy phù hợp với } \\
\text { môi trường số }\end{array}$ & \\
\hline \multirow[t]{2}{*}{$\begin{array}{l}\text { 3. Năng lực số và phát triển } \\
\text { ngôn ngữ }\end{array}$} & $\begin{array}{l}\text { 3.1. Đảm bảo sự phát triển ngôn ngữ của trẻ } \\
\text { trong môi trường số }\end{array}$ & \multirow[t]{2}{*}{$\begin{array}{l}\text { [22], [24] } \\
{[25],[31]}\end{array}$} \\
\hline & $\begin{array}{l}\text { 3.2. Thúc đẩy các kỹ năng ngôn ngữ thông qua } \\
\text { các sản phẩm số }\end{array}$ & \\
\hline \multirow[t]{4}{*}{$\begin{array}{l}\text { 4. Năng lực số và phát triển } \\
\text { cảm xúc - xã hội }\end{array}$} & $\begin{array}{l}\text { 4.1 Thúc đẩy năng lực giao tiếp trong môi } \\
\text { trường số }\end{array}$ & \multirow[t]{4}{*}{$\begin{array}{l}23],[25], \\
{[29],[31]}\end{array}$} \\
\hline & $\begin{array}{l}4.2 \text { Thúc đẩy năng lực cộng tác trong môi } \\
\text { trường số }\end{array}$ & \\
\hline & $\begin{array}{l}\text { 4.3 Tăng cường sự tham gia của gia đình trong } \\
\text { quá trình thụ đắc, phát triển các năng lực số }\end{array}$ & \\
\hline & $\begin{array}{l}\text { 4.4 Xây dựng, hình thành nhận thức về trách } \\
\text { nhiệm cá nhân trong môi trường số }\end{array}$ & \\
\hline
\end{tabular}




\begin{tabular}{|c|c|c|}
\hline & $\begin{array}{l}4.5 \text { Thúc đẩy năng lực sáng tạo trong môi } \\
\text { trường số }\end{array}$ & \\
\hline \multirow[t]{4}{*}{ 5. Cuộc sống số an toàn } & $\begin{array}{l}\text { 5.1. Đảm bảo tính riêng tư và bảo mật trong } \\
\text { môi trường số }\end{array}$ & {$[26],[30]$} \\
\hline & $\begin{array}{l}\text { 5.2. Đảm bảo tính an toàn trong việc sử dụng } \\
\text { các thiết bị số }\end{array}$ & {$[27] .[30]$} \\
\hline & 5.3. Những nội dung được khuyến khích & \multirow[t]{2}{*}[28]{,$[30]$} \\
\hline & $\begin{array}{l}\text { 5.4. Những nội dung không được khuyến } \\
\text { khích }\end{array}$ & \\
\hline \multirow[t]{3}{*}{$\begin{array}{l}\text { 6. Công bằng và bình đẳng } \\
\text { trong phát triển năng lực số }\end{array}$} & $\begin{array}{l}\text { 6.1. Giảm thiểu khoảng cách về khả năng tiếp } \\
\text { cận các thiết bị công nghệ }\end{array}$ & \multirow[t]{3}{*}{ [26], [28] } \\
\hline & $\begin{array}{l}\text { 6.2. Giảm thiểu khoảng cách về khả năng tiếp } \\
\text { cận các nội dung số }\end{array}$ & \\
\hline & $\begin{array}{l}\text { 6.3. Tăng cường năng lực sử dụng hiệu quả } \\
\text { các thiết bị và nội dung số }\end{array}$ & \\
\hline
\end{tabular}

Lưu ý:

- Các mục tiêu phát triển không chỉ hướng đến việc sử dụng các thiết bị công nghệ mà còn là về việc hạn chế như thế nào để phù hợp với các tiêu chuẩn.

- Lưu ý về các tiêu chuẩn khác nhau cho các loại hình thiết bị khác nhau. 


\section{TÀI LIỆU THAM KHẢO:}

1. Kabali HK, Irigoyen MM, Nunez-Davis R, et al. Exposure and use of mobile devices by young children. Pediatrics. 2015;136(6):1044-1050

2. Gilmore, J., Knickmeyer, R. \& Gao, W. Imaging structural and functional brain development in early childhood. Nat Rev Neurosci 19,123-137 (2018). https://doi.org/10.1038/nrn.2018.1

3. UNESCO Institute for Statistics. A Global Framework of Reference on Digital Literacy Skills for Indicator 4.4.2. 2018. UNESCO

4. UNESCO Education Sector. Conference on Digital Citizenship Education in Asia-Pacigic. 2017. UNESCO

5. American Academy of Pediatrics. 2016. Media and young minds.

6. Cox R, Skouteris H, Rutherford L, Fuller-Tyszkiewicz M, Dell' Aquila D, Hardy LL. Television viewing, television content, food intake, physical activity and body mass index: a cross-sectional study of preschool children aged 2-6 years. Health Promot J Austr. 2012;23(1):58-62

7. Taveras EM, Gillman MW, Kleinman KP, Rich-Edwards JW, Rifas-Shiman SL. Reducing racial/ethnic disparities in childhood obesity: the role of early life risk factors. JAMA Pediatr. 2013;167(8):731-738

8. Suglia SF, Duarte CS, Chambers EC, Boynton-Jarrett R. Social and behavioral risk factors for obesity in early childhood. J Dev Behav Pediatr. 2013;34(8):549-556

9. Vijakkhana N;Wilaisakditipakorn $\mathrm{T}$;Ruedeekhajorn $\mathrm{K}$;Pruksananonda C;Chonchaiya W;. (n.d.). Evening media exposure reduces night-time sleep. https://pubmed.ncbi.nlm.nih.gov/25521612/

10. Garrison MM, Liekweg K, Christakis DA. Media use and child sleep: the impact of content, timing, and environment. Pediatrics. 2011;128(1):29-35

11. Salti R, Tarquini R, Stagi S, et al. Age-dependent association of exposure to television screen with children's urinary melatonin excretion? Neuroendocrinol Lett. 2006;27(12):73-80

12. World Health Organization. Guidelines on physical activity, sedentary behaviour and sleep $\begin{array}{llllll}\text { for children under } 5 \text { years of age. } & 2019 .\end{array}$ 
https://apps.who.int/iris/bitstream/handle/10665/311664/9789241550536-

eng.pdf? sequence $=1 \&$ is Allowed $=\mathrm{y}$

13. Hatch, K.E. 2011. Determining the Effects of Technology on Children. Senior Honors Projects. Papers 260. http://digitalcommons.uri.edu/srhonorsprog/260

14. Wooldridge, M. B., \& Shapka, J. (2012). Playing with technology: Mother-toddler interaction scores lower during play with electronic toys. Journal of Applied Developmental Psychology, 33(5), 211-218. doi:10.1016/j.appdev.2012.05.005

15. Bergen, D., Hutchinson, K., Nolan, J. T., \& Weber, D. (2009). Effects of Infant-Parent Play With a Technology-Enhanced Toy: Affordance-Related Actions and Communicative Interactions. Journal of Research in Childhood Education, 24(1), 1-17. doi:10.1080/02568540903439342

16. Mcpake, J., Plowman, L., \& Stephen, C. (2012). Pre-school children creating and communicating with digital technologies in the home. Br J Educ Technol British Journal of Educational Technology, 44(3), 421-431. doi:10.1111/j.1467-8535.2012.01323.x

17. Shields, M. \& Behrman, R. E. 2000. Children and Computer Technology: Analysis and Recommendations. Journal Issue: Children and Computer Technology, (Online), Volume 10 Number 2 Fall/Winter, http://www.futureofchildren.org/publications/docs/10_02_FulJournal.pdf.

18. Walters, J. 2015. Smartphones Damage Social Development. Toddler Brains Research. https://www.theguardian.com/technology/2015/feb/01/toddlerbrainsresearchsmartphonesdamage-social-development

19. Livingstone, Sonia, Giovanna Mascheroni and Elisabeth Staksrud, 'Developing a Framework for Researching Children's Online Risks and Opportunities in Europe', $E U$ Kids Online, London, 2015.

20. Chassiakos, Y. L. R., Radesky, J., Christakis, D., Moreno, M. A., \& Cross, C. (2016). Children and adolescents and digital media. Pediatrics, 138(5), e20162593. https://doi.org/10.1542/peds.2016-2593

21. American Academy of Pediatrics. (2016, October). American Academy of Pediatrics Announces New Recommendations for Children's Media, American Academy of Pediatrics. https://services.aap.org/en/news-room/news-releases/aap/2016/aap-announcesnew-recommendations-for-media-use/ 
22. Vulchanova, M., Baggio, G., Cangelosi, A., \& Smith, L. (2017). Language Development in the Digital Age. Frontiers in Human Neuroscience, 11, 447. https://doi.org/10.3389/fnhum.2017.00447

23. Brown, J., Winsor, D. L., \& Blake, S. (2012). Technology and social-emotional development in the early childhood environments. In Child development and the use of technology: Perspectives, applications and experiences (pp. 112-128). IGI Global. https://doi.org/10.4018/978-1-61350-317-1.ch006

24. Cooper, L. Z. (2005). Developmentally appropriate digital environments for young children. Library trends, 54(2), 286-302. https://doi.org/10.1353/lib.2006.0014

25. Marinelli, S. (2017). A Child's World: How Electronics Influence Children's Language, Physical, and Social Emotional Development. [Honors Theses, Assumption University]. https://digitalcommons.assumption.edu/honorstheses/23

26. Keeley, B., \& Little, C. (2017). The State of the Worlds Children 2017: Children in a Digital World. UNICEF. https://www.unicef.org/publications/files/SOWC_2017_ENG_WEB.pdf

27. American Psychological Association. (2019). Digital guidelines: Promoting healthy technology use for children, American Psychological Association https://www.apa.org/topics/healthy-technology-use-children

28. National Association for the Education of Young Children. (2012). Technology and interactive media as tools in early childhood programs serving children from birth through age 8. Spotlight on young children and technology, 61-70. https://www.naeyc.org/sites/default/files/globallyshared/downloads/PDFs/resources/topics/PS_technology_WEB.pdf

29. Quesenberry, A., Mustian, A., \& Clark-Bischke, C. (2016). Preschool and Kindergarten: Tuning in: Strategies for Incorporating Technology Into Social Skills Instruction in Preschool and Kindergarten. YC Young Children, 71(1), 74-81. http://www.jstor.org/stable/ycyoungchildren.71.1.74

30. Noah, Z. \& Adrienne, L. (2020). Children's Safety and Privacy in the Digital Age. CSA Group. https://www.csagroup.org/wp-content/uploads/CSA-Group-Research-ChildrensSafety-and-Privacy-in-the-Digital-Age.pdf 
31. Office of Educational Technology. (n.d.). Guiding Principles for Use of Technology with Early Learners, Office of Educational Technology.

https://tech.ed.gov/earlylearning/principles/\#: :text=Developmentally\%20appropriate\%2 Ouse \%20of\%20technology,in\%20fun\%20and\%20challenging\%20activities.

32. Hoang, A. D., Pham, H. H., Nguyen, Y. C., Vuong, Q. H., Dam, M. Q., Tran, T., \& Nguyen, T. T. (2020). Introducing a tool to gauge curriculum quality under Sustainable Development Goal 4: The case of primary schools in Vietnam. International Review of Education, 66(4), 457-485.

33. Ho, M. T., La, V. P., Nguyen, M. H., Pham, T. H., Vuong, T. T., Vuong, H. M., ... \& Vuong, Q. H. (2020). An analytical view on STEM education and outcomes: Examples of the social gap and gender disparity in Vietnam. Children and Youth Services Review, 119, 105650.

34. Le, A. V., Do, D. L., Pham, D. Q., Hoang, P. H., Duong, T. H., Nguyen, H. N., ... \& Vuong, Q. H. (2019). Exploration of youth's digital competencies: a dataset in the educational context of Vietnam. Data, 4(2), 69. 